\title{
MOTION OF LEVEL SETS BY MEAN CURVATURE. II
}

\author{
L. C. EVANS AND J. SPRUCK
}

\begin{abstract}
We give a new proof of short time existence for the classical motion by mean curvature of a smooth hypersurface. Our method consists in studying a fully nonlinear uniformly parabolic equation satisfied by the signed distance function to the surface
\end{abstract}

\section{INTRODUCTION}

We present in this paper a new, elementary, and fairly concise proof of short time existence for the classical motion of a smooth hypersurface evolving according to its mean curvature. In this problem we are given initially a smooth connected hypersurface $\Gamma_{0}$, which is the boundary of a bounded open set $U \subset \mathbf{R}^{n}$. We then allow $\Gamma_{0}$ to evolve in time into a family of surfaces $\left\{\Gamma_{t}\right\}_{t>0}$ by moving each point on $\Gamma_{t}(t \geq 0)$ at a velocity equal to $(n-1)$ times the mean curvature vector at that point. Our intent is to verify that for small times at least, the classical motion as thus envisioned in fact exists and is unique. This assertion was first proved by Gage and Hamilton [4], based on the the work of Hamilton [5], and we discuss below the relation of our work to theirs.

This is a companion to our paper [3], wherein we defined and then studied a generalized notion of evolution via mean curvature, existing for all times and agreeing with the classical motion, if and so long as the latter exists. This generalized evolution is constructed in [3] by first building an appropriately defined unique weak solution of the PDE

$$
\begin{aligned}
& u_{t}=\left(\delta_{i j}-\frac{u_{x_{i}} u_{x_{j}}}{|D u|^{2}}\right) u_{x_{i} x_{j}} \text { in } \mathbf{R}^{n} \times(0, \infty), \\
& u=g \text { on } \mathbf{R}^{n} \times\{t=0\} .
\end{aligned}
$$

Equation (1.1) says that each level set of $u$ evolves according to its mean curvature, at least in regions where $u$ is smooth and $|D u| \neq 0$. Given then $\Gamma_{0}$ as above, we select a smooth function $g: \mathbf{R}^{n} \rightarrow \mathbf{R}$ for which

$$
\Gamma_{0}=\left\{x \in \mathbf{R}^{n} \mid g(x)=0\right\} .
$$

We next write

$$
\Gamma_{t} \equiv\left\{x \in \mathbf{R}^{n} \mid u(x, t)=0\right\} \quad(t \geq 0),
$$

Received by the editors January 4, 1990.

1980 Mathematics Subject Classification (1985 Revision). Primary 35K55, 53A10.

The first author was supported in part by NSF grant DMS-86-01532, and the second was supported in part by NSF grant DMS-85-01952 and DOE grant DE-FG02-86ER250125. 
and call the family of sets $\left\{\Gamma_{t}\right\}_{t \geq 0}$ so defined the generalized evolutions by mean curvature starting from $\Gamma_{0}$. The rest of [3] is devoted to deducing from the PDE (1.1), (1.2) various elementary geometric properties of the flow $\Gamma_{0} \mapsto \Gamma_{t}$ $(t \geq 0)$. A very similar investigation of this and more complicated problems has been independently developed by Chen, Giga, and Goto [2].

In this paper we return again to the idea of a studying a nonlinear PDE, a level set of whose solution evolves via mean curvature. Our idea is first to assume that $\Gamma_{0}$ develops by classical mean curvature motion, at least for times $0 \leq t \leq t_{0}$, and then to derive the PDE verified by $d$, the signed distance function to the surface at each time. This turns out to be a fully nonlinear, uniformly parabolic equation: see (1.9) below. Next we construct for short times a smooth solution of this equation, subject to nonlinear boundary conditions in an appropriate region, and then finally verify that our solution is in fact the signed distance function to a family of smooth surfaces evolving from $\Gamma_{0}$ by mean curvature motion.

We proceed now to the heuristic derivation of our PDE. Suppose therefore we are given the smooth hypersurface $\Gamma_{0}=\partial U$ as above, a time $t_{0}>0$, and a classical evolution $\left\{\Gamma_{t}\right\}_{0 \leq t \leq t_{0}}$ of surfaces developing from $\Gamma_{0}$ by mean curvature flow. Then for each time $t$ in $\left[0, t_{0}\right], \Gamma_{t}$ is the smooth boundary of a bounded open set $U_{t}$, which is diffeomorphic to $U=U_{0}$. Define the signed distance function

$$
d(x, t) \equiv\left\{\begin{aligned}
\operatorname{dist}\left(x, \Gamma_{t}\right) & \left(x \in \mathbf{R}^{n}-\bar{U}_{t}\right), \\
-\operatorname{dist}\left(x, \Gamma_{t}\right) & \left(x \in U_{t}\right)
\end{aligned}\right.
$$

for $x \in \mathbf{R}^{n}, 0 \leq t \leq t_{0}$. By assumption $\Gamma \equiv \bigcup_{0 \leq t \leq t_{0}} \Gamma_{t} \times\{t\}$ is smooth, and thus $d$ is smooth in

$$
Q^{+} \equiv\left\{(x, t) \mid 0<t<t_{0}, 0<d(x, t)<\delta_{0}\right\}
$$

and in

$$
Q^{-}=\left\{(x, t) \mid 0<t<t_{0},-\delta_{0}<d(x, t)<0\right\}
$$

for $\delta_{0}>0$ small enough. Fix any point $(x, t) \in Q^{+}$. Then provided $\delta_{0}>0$ is sufficiently small, there exists a unique point $y \in \Gamma_{t}$ for which $d(x, t)=|x-y|$. Let $\nu=D d$ be the smooth unit normal vector field pointing from $\Gamma$ into $Q^{+}$. As $\left\{\Gamma_{t}\right\}_{0 \leq t \leq t_{0}}$ moves via mean curvature, we have

$$
d_{t}(x, t)=\operatorname{div}(\nu)(y, t) .
$$

On the other hand the (unordered) eigenvalues of the matrix $D^{2} d(x, t)$ are

$$
\begin{array}{ll}
\lambda_{i} & =\lambda_{i}\left(D^{2} d(x, t)\right)=-\kappa_{i} /\left(1-\kappa_{i} d(x, t)\right) \quad(1 \leq i \leq n-1) \\
\lambda_{n} & =\lambda_{n}\left(D^{2} d(x, t)\right)=0,
\end{array}
$$

$\left\{\kappa_{1}, \ldots, \kappa_{n-1}\right\}$ denoting the principal curvatures of $\Gamma_{t}$ at $y$, computed with respect to the normal field $\nu$. Inverting (1.6) we compute

$$
\kappa_{i}=\frac{\lambda_{i}}{\lambda_{i} d(x, t)-1} \quad(1 \leq i \leq n-1) .
$$

Since

$$
\operatorname{div}(\nu)=-\left(\kappa_{1}+\cdots+\kappa_{n-1}\right)
$$


we deduce finally

$$
d_{t}(x, t)=f\left(\lambda_{1}\left(D^{2} d(x, t)\right), \ldots, \lambda_{n}\left(D^{2} d(x, t)\right), d(x, t)\right)
$$

for

$$
f\left(\lambda_{1}, \ldots, \lambda_{n}, z\right) \equiv \sum_{i=1}^{n} \frac{\lambda_{i}}{1-\lambda_{i} z} .
$$

The same formula results if $(x, t) \in Q^{-}$.

Now our PDE (1.7) has the general form

$$
d_{t}=F\left(D^{2} d, d\right)
$$

and, as $f$ is symmetric in the variables $\lambda_{1}, \ldots, \lambda_{n}, F$ is smooth. Since

$$
\frac{\partial f}{\partial \lambda_{i}}=\frac{1}{\left(1-\lambda_{i} d\right)^{2}}>0 \quad(1 \leq i \leq n),
$$

equation (1.9) is uniformly parabolic. See Caffarelli, Nirenberg, and Spruck [1] for more details.

Our plan is to study directly the PDE (1.7), (1.8). For this suppose now $\Gamma_{0}$ is the smooth connected boundary of a bounded open set $U \subset \mathbf{R}^{n}$, and let

$$
g(x) \equiv\left\{\begin{aligned}
\operatorname{dist}\left(x, \Gamma_{0}\right) & \left(x \in \mathbf{R}^{n}-\bar{U}\right), \\
-\operatorname{dist}\left(x, \Gamma_{0}\right) & (x \in U)
\end{aligned}\right.
$$

be the signed distance function. Fix then $\delta_{0}>0$ so small that $g$ is smapth within

$$
V \equiv\left\{x \in \mathbf{R}^{n} \mid-\delta_{0}<g<\delta_{0}\right\}
$$

and write

$$
Q \equiv V \times\left(0, t_{0}\right), \quad \Sigma \equiv \partial V \times\left[0, t_{0}\right] .
$$

In $\S 2$ we construct a smooth solution $v$ to the PDE

$$
\begin{cases}v_{t}=F\left(D^{2} v, v\right) & \text { in } Q \\ |D v|^{2}=1 & \text { on } \Sigma, \\ v=g & \text { on } V \times\{t=0\}\end{cases}
$$

for some small time $t_{0}>0$, our argument being in effect a special case of the Inverse Function Theorem. We then verify in $\S 3$ that

$$
|D v|^{2}=1 \text { in } Q \text {. }
$$

Setting

$$
\Gamma_{t} \equiv\{x \in V \mid v(x, t)=0\} \quad\left(0 \leq t \leq t_{0}\right),
$$

we deduce from (1.13) and (1.14) that $\left\{\Gamma_{t}\right\}_{0 \leq t \leq t_{0}}$ is a smooth evolution gayerned by mean curvature and $v=d$ is the corresponding signed distance fifnetion.

Section 4 utilizes the PDE (1.7), (1.8) to prove an "instantaneous tepring apart" assertion for close-by evolving surfaces. Some consequences are discussed in $[3, \S 8]$.

As noted, Gage and Hamilton in $[4, \S 2]$ have previously established the shorttime existence of the classical mean curvature evolution by studying the ddgenerate parabolic system describing the parametrized surface. As this system is 
degenerately parabolic, they must appeal to a general theorem of Hamilton [5, Theorem 5.1] which employs fairly complicated techniques related to the NashMoser Implicit Function Theorem. Our methods are simpler.

\section{SOLVING THE NONLINEAR PDE}

Our goal in this section is to construct a smooth solution of the PDE (1.13) for $\delta_{0}>0$ and $t_{0}>0$ small enough. Let us first of all select $\delta_{0}$ so small that

$$
M \delta_{0} \leq \frac{1}{4}
$$

for

$$
M \equiv \max _{V}\left|D^{2} g\right|
$$

Set

$$
G \equiv\left\{R \in S^{n \times n}, z \in \mathbf{R}|| z\left|<\delta_{0},\right| R \mid<2 M\right\} .
$$

Then since (2.1) implies

$$
\left|\lambda_{i}(R) z\right| \leq|R||z| \leq \frac{1}{2} \quad(1 \leq i \leq n)
$$

if $(R, z) \in G$,

$$
F(R, z)=f\left(\lambda_{1}(R), \ldots, \lambda_{n}(R), z\right)=\sum_{i=1}^{n} \frac{\lambda_{i}(R)}{1-\lambda_{i}(R) z}
$$

is defined and smooth on $G$. Arbitrarily extend $F$ off $G$ to be smooth on all of $S^{n \times n} \times \mathbf{R}$, with $|F|,|D F|,\left|D^{2} F\right|$ bounded.

We now check that our PDE is uniformly parabolic near $g$.

Lemma 2.1. Then exists a constant $\theta>0$ so that

$$
\frac{\partial F}{\partial r_{i j}}(R, z) \xi_{i} \xi_{j} \geq \theta|\xi|^{2} \quad\left(\xi \in \mathbf{R}^{n}\right)
$$

in $V$ for each $(R, z) \in G$.

Proof. Fix $\xi \in \mathbf{R}^{n}$ and choose then $t>0$ so small that $(R+t \xi \otimes \xi, z) \in G$. Using Courant's minimax characterization we deduce that the eigenvalues can be ordered so that

$$
\lambda_{k}(R+t \xi \otimes \xi) \geq \lambda_{k}(R) \quad(k=1, \ldots, n) .
$$

Consequently

$$
\begin{aligned}
F(R+ & t \xi \otimes \xi, z)-F(R, z) \\
& =f\left(\ldots, \lambda_{k}(R+t \xi \otimes \xi), \ldots, z\right)-f\left(\ldots, \lambda_{k}(R), \ldots, z\right) \\
& =\sum_{i=1}^{n} \int_{0}^{1} \frac{\partial f}{\partial \lambda_{k}}\left(\ldots, s \lambda_{k}(R+t \xi \otimes \xi)\right. \\
& \left.\quad+(1-s) \lambda_{k}(R), \ldots, z\right) d s\left[\lambda_{k}(R+t \xi \otimes \xi)-\lambda_{k}(R)\right] \\
& \geq \theta \operatorname{trace}(R+t \xi \otimes \xi-R)
\end{aligned}
$$

for some $\theta>0$ by (1.10). Dividing by $t>0$ and sending $t \rightarrow 0^{+}$, we arrive at inequality (2.4).

We will seek our solution $v$ of the PDE (1.13) in the form

$$
v=g+t h+w,
$$


where

$$
h \equiv F\left(D^{2} g, g\right)
$$

Then plugging into (1.13) we compute

for

$$
w_{t}-a_{i j} w_{x_{i} x_{j}}+c w=A\left(D^{2} w, w, x, t\right) \text { in } Q
$$

and

$$
a_{i j} \equiv \frac{\partial F}{\partial r_{i j}}\left(D^{2} g, g\right), \quad c \equiv \frac{-\partial F}{\partial z}\left(D^{2} g, g\right)
$$

$$
\begin{aligned}
A(R, z, x, t) \equiv & F\left(D^{2} g+t D^{2} h+R, g+t h+z\right) \\
& -F\left(D^{2} g, g\right)-\frac{\partial F}{\partial r_{i j}}\left(D^{2} g, g\right) r_{i j}-\frac{\partial F}{\partial z}\left(D^{2} g, g\right) z .
\end{aligned}
$$

We next insert (2.5) into the nonlinear boundary condition from the PDE (1.13). Recalling that $|D g|^{2}=1$ and $D g$ is normal to $\partial V$ we discover

$$
\frac{\partial w}{\partial \nu}=a(D w, x, t) \text { on } \Sigma \text {, }
$$

where $\nu$ is the outer unit normal vector field along $\Sigma$ and

$$
a(p, x, t) \equiv \begin{cases}-\frac{1}{2}|t D h+p|^{2}-t \frac{\partial h}{\partial \nu} & \text { on }\left\{d=\delta_{0}\right\} \\ \frac{1}{2}|t D h+p|^{2}-t \frac{\partial h}{\partial \nu} & \text { on }\left\{d=-\delta_{0}\right\}\end{cases}
$$

Combining everything above, we hereafter seek a smooth function $w$ satisfying

$$
\begin{cases}w_{t}-a_{i j} w_{x_{i} x_{j}}+c w=A\left(D^{2} w, w, x, t\right) & \text { in } Q \\ \frac{\partial w}{\partial \nu}=a(D w, x, t) & \text { on } \Sigma, \\ w=0 & \text { on } V \times\{t=0\} .\end{cases}
$$

We intend to solve (2.10) by finding a fixed point of the mapping $T$ defined by inserting a given function into the nonlinear terms $A, a$ and solving the resulting linear PDE.

We will work in certain standard parabolic-type Hölder spaces. Fix $0<$ $\alpha<1$. We define several norms, following Ladyzhenskaja, Solonnikov, and Ural'tseva [6, pp. 7-8]. Set

$$
\begin{aligned}
& |u|^{(0)} \equiv \sup \{|u(x, t)| \mid(x, t) \in Q\} \\
& |u|^{(1)} \equiv|u|^{(0)}+|D u|^{(0)} \\
& |u|^{(2)} \equiv|u|^{(1)}+\left|D^{2} u\right|^{(0)}+\left|u_{t}\right|^{(0)} \\
& \langle u\rangle_{x}^{(\beta)} \equiv \sup \left\{\frac{|u(x, t)-u(y, t)|}{|x-y|^{\beta}} \mid(x, t),(y, t) \in Q, x \neq y\right\}, \\
& \langle u\rangle_{t}^{(\beta)} \equiv \sup \left\{\frac{|u(x, t)-u(x, s)|}{|t-s|^{\beta}} \mid(x, t),(x, s) \in Q, t \neq s\right\},
\end{aligned}
$$

for $0<\beta<1$. Then

$$
\begin{aligned}
\|u\|_{C^{\alpha, \frac{\alpha}{2}}(\bar{Q})} \equiv|u|^{(0)}+\langle u\rangle_{x}^{(\alpha)}+\langle u\rangle_{t}^{\left(\frac{\alpha}{2}\right)} \\
\|u\|_{C^{1+\alpha, \frac{1+\alpha}{2}}(\bar{Q})} \equiv|u|^{(1)}+\langle D u\rangle_{x}^{(\alpha)}+\langle u\rangle_{t}^{\left(\frac{1+\alpha}{2}\right)}+\langle D u\rangle_{t}^{\left(\frac{\alpha}{2}\right)} \\
\|u\|_{C^{2+\alpha, \frac{2+\alpha}{2}}(\bar{Q})} \equiv|u|^{(2)}+\langle D u\rangle_{t}^{\left(\frac{1+\alpha}{2}\right)}+\left\langle D^{2} u\right\rangle_{x}^{(\alpha)}+\left\langle u_{t}\right\rangle_{x}^{(\alpha)}+\left\langle D^{2} u\right\rangle_{t}^{\left(\frac{\alpha}{2}\right)}+\left\langle u_{t}\right\rangle_{t}^{\left(\frac{\alpha}{2}\right)}
\end{aligned}
$$


Note

Finally define

$$
\|D u\|_{C^{1+\alpha, \frac{1+\alpha}{2}}(\bar{Q})},\left\|D^{2} u\right\|_{C^{\alpha, \frac{\alpha}{2}}(\bar{Q})},\left\|u_{t}\right\|_{C^{\alpha, \frac{\alpha}{2}}(\bar{Q})} \leq\|u\|_{C^{2+\alpha}, \frac{2+\alpha}{2}(\bar{Q})} .
$$

$$
\|u\|_{C^{1+\alpha, \frac{1+\alpha}{2}(\Sigma)}} \equiv \inf \left\{\|v\|_{C^{1+\alpha, \frac{1+\alpha}{2}}(\bar{Q})} \mid v=u \text { on } \Sigma\right\} .
$$

We consider now the linear, uniformly parabolic PDE

$$
\begin{cases}w_{t}-a_{i j} w_{x_{i} x_{j}}+c w=B & \text { in } Q \\ \frac{\partial w}{\partial \nu}=b & \text { on } \Sigma \\ w=0 & \text { on } V \times\{t=0\}\end{cases}
$$

Suppose as well

$$
b=0 \quad \text { on } \partial V \times\{t=0\} .
$$

Lemma 2.2. Assume $B \in C^{\alpha, \frac{\alpha}{2}}(\bar{Q}), b \in C^{1+\alpha, \frac{1+\alpha}{2}}(\Sigma)$, and the zeroth order compatibility condition (2.12) holds. Then there exists a unique solution $w \in$ $C^{2+\alpha}, \frac{2+\alpha}{2}(\bar{Q})$ of the PDE (2.11), with the estimate

$$
\|w\|_{C^{2+\alpha}, \frac{2+\alpha}{2}(\bar{Q})} \leq C\left(\|B\|_{C^{\alpha, \frac{\alpha}{2}}(\bar{Q})}+\|b\|_{C^{1+\alpha, \frac{1+\alpha}{2}}(\Sigma)}\right) .
$$

The constant $C$ does not depend on $t_{0}$.

Proof. Except for the last assertion, this is Theorem IV.5.3 in Ladyzhenskaja, Solonnikov, Ural'tseva [6, pp. 320-321]. To obtain the statement that the constant $C$ remains bounded even for small $0<t_{0}<1$, we define

$$
\begin{aligned}
\tilde{Q} & =V \times(0,1), \quad \tilde{\Sigma}=\partial V \times(0,1), \\
\tilde{B}(x, t) & = \begin{cases}B(x, t) & \text { if } 0 \leq t \leq t_{0}, \\
B\left(x, t_{0}\right) & \text { if } t_{0} \leq t \leq 1,\end{cases} \\
\tilde{b}(x, t) & = \begin{cases}b(x, t) & \text { if } 0 \leq t \leq t_{0}, \\
b\left(x, t_{0}\right) & \text { if } t_{0} \leq t \leq 1 .\end{cases}
\end{aligned}
$$

Then

$$
\|\widetilde{B}\|_{C^{\alpha, \frac{\alpha}{2}(\widetilde{Q})}}=\|B\|_{C^{\alpha, \frac{\alpha}{2}(Q)}}, \quad\|\tilde{b}\|_{C^{1+\alpha, \frac{1+\alpha}{2}(\widetilde{\Sigma})}}=\|b\|_{C^{1+\alpha, \frac{1+\alpha}{2}}(\Sigma)} .
$$

Let $\tilde{w} \in C^{2+\alpha} \frac{2+\alpha}{2}(\widetilde{Q})$ solve the $\operatorname{PDE}(2.11)$ with $\widetilde{Q}, \widetilde{\Sigma}, \widetilde{B}, \tilde{b}$ replacing $Q$, $\Sigma, B, b$. The estimate $(2.13)$ then is valid for $\tilde{w}$ with $\widetilde{Q}, \widetilde{\Sigma}$ replacing $Q, \Sigma$. But $\tilde{w}=w$ on $Q$, by uniqueness for parabolic equations.

We will henceforth work in the Banach space

$$
X \equiv\left\{w \in C^{2+\alpha}, \frac{2+\alpha}{2}(\bar{Q}) \mid w=0 \text { on } V \times\{t=0\}\right\}
$$

Givien $\hat{w} \in X$, we set

$$
\left\{\begin{array}{l}
B(x, t) \equiv A\left(D^{2} \hat{w}, \hat{w}, x, t\right) \\
b(x, t) \equiv a(D \hat{w}, x, t)
\end{array}\right.
$$

for $A, a$ defined by (2.8), (2.9). Now write $T(\hat{w})=w$, when $w \in X$ solves the linear PDE (2.11), for $B, b$ as in (2.14). We seek a fixed point of the mapping $T: X \rightarrow X$. Give $r_{0}>0$, set

$$
Y \equiv\left\{w \in X \mid\|w\|_{C^{2+\alpha}, \frac{2+\alpha}{2}(Q)} \leq r_{0}\right\}
$$


Lemma 2.3. If $t_{0}, r_{0}>0$ are sufficiently small, then

$$
T: Y \rightarrow Y \text {. }
$$

Proof. 1. Choose any function $\hat{w} \in Y$, define $B, b$ by (2.14), and let $w \in X$ solve (2.11). We must show the inequality

$$
\|\hat{w}\|_{C^{2+\alpha}, \frac{2+\alpha}{2}(\bar{Q})} \leq r_{0}
$$

implies

$$
\|w\|_{C^{2+\alpha}, \frac{2+\alpha}{2}(\bar{Q})} \leq r_{0},
$$

provided $r_{0}, t_{0}>0$ are adjusted sufficiently small.

2. Now (2.8) implies

$$
\begin{aligned}
A(R, z, x, t)= & t \frac{\partial F}{\partial r_{i j}}\left(D^{2} g, g\right) h_{x_{i} x_{j}}+t \frac{\partial F}{\partial z}\left(D^{2} g, g\right) h \\
& +\int_{0}^{1}(1-s) \frac{\partial^{2} F}{\partial r_{i j} \partial r_{k l}}\left(D^{2} g+s t D^{2} h+s R, g+s t h+s z\right) d s \\
& \times\left(t h_{x_{i} x_{j}}+r_{i j}\right)\left(t h_{x_{k} x_{l}}+r_{k l}\right) \\
& +2 \int_{0}^{1}(1-s) \frac{\partial^{2} F}{\partial r_{i j} \partial z}\left(D^{2} g+s t D^{2} h+s R, g+s t h+s z\right) d s \\
& \times\left(t h_{x_{i} x_{j}}+r_{i j}\right)(t h+z) \\
& +\int_{0}^{1}(1-s) \frac{\partial^{2} F}{\partial z^{2}}\left(D^{2} g+s t D^{2} h+s R, g+s t h+s z\right) d s \\
& \times(t h+z)^{2} .
\end{aligned}
$$

Recall also that

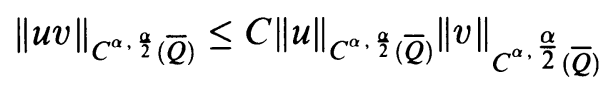

for all $u, v \in C^{\alpha, \frac{\alpha}{2}}(\bar{Q})$, and

$$
\|t\|_{C^{\alpha, \frac{\alpha}{2}(\bar{Q})}} \leq C t_{0}^{1-\frac{\alpha}{2}} .
$$

Then (2.14), (2.18), (2.20) imply

$$
\|B\|_{C^{\alpha, \frac{\alpha}{2}(\bar{Q})}} \leq C\left(r_{0}^{2}+t_{0}^{1-\frac{\alpha}{2}}\right) .
$$

Similarly formula (2.9) implies

$$
\|b\|_{C^{1+\alpha, \frac{1+\alpha}{2}(\Sigma)}} \leq C\left(r_{0}^{2}+t_{0}^{\frac{1-\alpha}{2}}\right)
$$

3. Inserting estimates (2.21), (2.22) into (2.13) we discover

$$
\|w\|_{C^{2+\alpha}, \frac{2+\alpha}{2}(\bar{Q})} \leq C\left(r_{0}^{2}+t_{0}^{\frac{1-\alpha}{2}}\right) \text {, }
$$

the constant $C$ independent of $r_{0}$ and $t_{0}$. Let $r_{0} \leq \frac{1}{2 C}$. Then (2.23) gives

$$
\|w\|_{C^{2+\alpha, \frac{2+\alpha}{2}(\bar{Q})}} \leq \frac{r_{0}}{2}+C t_{0}^{\frac{1-\alpha}{2}} \leq r_{0}
$$

if $t_{0}=t_{0}\left(r_{0}\right)$ is small enough. This verifies inequality (2.17).

Finally we check that $T: Y \rightarrow Y$ is a strict contraction. 
Lemma 2.4. If $r_{0}, t_{0}>0$ are small enough,

$$
\left\|T\left(\hat{w}_{1}\right)-T\left(\hat{w}_{2}\right)\right\|_{C^{2+\alpha}, \frac{2+\alpha}{2}(\bar{Q})} \leq \frac{1}{2}\left\|\hat{w}_{1}-\hat{w}_{2}\right\|_{C^{2+\alpha, \frac{2+\alpha}{2}}(\bar{Q})}
$$

for all $\hat{w}_{1}, \hat{w}_{2} \in Y$.

Proof. 1. Choose $\hat{w}_{1}, \hat{w}_{2} \in Y$; then

$$
\left\|\hat{w}_{1}, w_{2}\right\|_{C^{2+\alpha}, \frac{2+\alpha}{2}(\bar{Q})} \leq r_{0} .
$$

Set

$$
\begin{cases}B_{1}(x, t) \equiv A\left(D^{2} \hat{w}_{1}, \hat{w}_{1}, x, t\right), & B_{2}(x, t) \equiv A\left(D^{2} \hat{w}_{2}, \hat{w}_{2}, x, t\right), \\ b_{1}(x, t) \equiv a\left(D \hat{w}_{1}, x, t\right), & b_{2}(x, t) \equiv a\left(D \hat{w}_{2}, x, t\right),\end{cases}
$$

and write $w_{1} \equiv T\left(\hat{w}_{1}\right), w_{2} \equiv T\left(\hat{w}_{2}\right)$. Thus $w_{1}$ solves the linear PDE (2.11) with $B_{1}, b_{1}$ replacing $B, b$, and $w_{2}$ solves (2.11) with $B_{2}, b_{2}$ replacing $B, b$. According to Lemma 2.2

$$
\left\|w_{1}-w_{2}\right\|_{C^{2+\alpha, \frac{2+\alpha}{2}(\bar{Q})}} \leq C\left(\left\|B_{1}-B_{2}\right\|_{C^{\alpha, \frac{\alpha}{2}}(\bar{Q})}+\left\|b_{1}-b_{2}\right\|_{C^{1+\alpha, \frac{1+\alpha}{2}(\Sigma)}}\right) .
$$

2. Recall that

$$
\begin{aligned}
& \|\Phi(u)-\Phi(v)\|_{C^{\alpha, \frac{\alpha}{2}}(\bar{Q})} \\
& \quad \leq C\left(\|u\|_{C^{\alpha, \frac{\alpha}{2}(\bar{Q})}}+\|v\|_{C^{\alpha, \frac{\alpha}{2}(\bar{Q})}}+1\right)\|u-v\|_{C^{\alpha, \frac{\alpha}{2}(\bar{Q})}}
\end{aligned}
$$

if $u, v \in C^{\alpha, \frac{\alpha}{2}}(\bar{Q})$ and $\Phi$ is smooth, with $D \Phi, D^{2} \Phi$ bounded.

Utilizing then formulas $(2.18)-(2.20),(2.27)$ we compute

$$
\left\|B_{1}-B_{2}\right\|_{C^{\alpha, \frac{\alpha}{2}}(\bar{Q})} \leq C\left(r_{0}+t_{0}^{1-\frac{\alpha}{2}}\right)\left\|\hat{w}_{1}-\hat{w}_{2}\right\|_{C^{2+\alpha}, \frac{2+\alpha}{2}(\bar{Q})} .
$$

Similarly

$$
\left\|b_{1}-b_{2}\right\|_{C^{1+\alpha, \frac{1+\alpha}{2}(\Sigma)}} \leq C\left(r_{0}+t_{0}^{\frac{1-\alpha}{2}}\right)\left\|\hat{w}_{1}-\hat{w}_{2}\right\|_{C^{2+\alpha, \frac{2+\alpha}{2}}(\bar{Q})} .
$$

Combining (2.26), (2.28), (2.29), we obtain estimate (2.24), provided $r_{0}, t_{0}>0$ are small.

We at last apply Banach's Fixed Point Theorem to establish

Theorem 2.5. If $\delta_{0}, t_{0}>0$ are sufficiently small, there exists a unique solution $v \in C^{2+\alpha}, \frac{2+\alpha}{2}(\bar{Q}) \cap C^{\infty}(Q)$ of the PDE (1.13).

In particular $\left(D^{2} v, v\right) \in G$ in $Q$.

\section{Motion OF THE ZERO LEVEL SET BY MEAN CURVATURE}

This section we devote to proving that the sets

$$
\Gamma_{t} \equiv\{x \in V \mid v(x, t)=0\} \quad\left(0 \leq t \leq t_{0}\right)
$$

are in fact smooth hypersurfaces evolving by mean curvature. 
Theorem 3.1. We have

$$
|D v|^{2}=1 \text { in } Q
$$

Proof. 1. Let $w \equiv|D v|^{2}-1 \in C^{1}(\bar{Q}) \cap C^{\infty}(Q)$. Then

$$
w=0 \text { on } \Sigma
$$

and

$$
w=0 \text { on } V \times\{t=0\},
$$

according to (1.11) and the PDE (1.13).

2. Differentiating (1.13) we compute

$$
v_{t x_{k}}=\frac{\partial F}{\partial r_{i j}}\left(D^{2} v, v\right) v_{x_{i} x_{j} x_{k}}+\frac{\partial F}{\partial z}\left(D^{2} v, v\right) v_{x_{k}} \quad(k=1, \ldots, n) .
$$

Thus

$$
\begin{aligned}
w_{t} & =2 v_{x_{k}} v_{x_{k} t} \\
& =2 \frac{\partial F}{\partial r_{i j}}\left(D^{2} v, v\right) v_{x_{k}} v_{x_{k} x_{i} x_{j}}+2 \frac{\partial F}{\partial z}\left(D^{2} v, v\right)|D v|^{2} \\
& =\frac{\partial F}{\partial r_{i j}}\left(D^{2} v, v\right) w_{x_{i} x_{j}}-2 \frac{\partial F}{\partial r_{i j}}\left(D^{2} v, v\right) v_{x_{k} x_{i}} v_{x_{k} x_{j}}+2 \frac{\partial F}{\partial z}\left(D^{2} v, v\right)|D v|^{2} .
\end{aligned}
$$

Now

$$
F\left(D^{2} v, v\right)=f\left(\ldots, \lambda_{i}\left(D^{2} v\right), \ldots, v\right) \text { in } Q
$$

Thus

$$
\frac{\partial F}{\partial z}\left(D^{2} v, v\right)=\frac{\partial f}{\partial z}\left(\ldots, \lambda_{i}\left(D^{2} v\right), \ldots, v\right)=\sum_{i=1}^{n} \frac{\lambda_{i}\left(D^{2} v\right)^{2}}{\left(1-\lambda_{i}\left(D^{2} v\right) v\right)^{2}}
$$

On the other hand (see Caffarelli, Nirenberg, and Spruck [1])

$$
\begin{aligned}
\frac{\partial F}{\partial r_{i j}}\left(D^{2} v\right) v_{x_{k} x_{i}} v_{x_{k} x_{j}} & =\sum_{i=1}^{n} \frac{\partial f}{\partial \lambda_{i}}\left(\ldots, \lambda_{i}\left(D^{2} v\right), \ldots, v\right) \lambda_{i}\left(D^{2} v\right)^{2} \\
& =\sum_{i=1}^{n} \frac{\lambda_{i}\left(D^{2} v\right)^{2}}{\left(1-\lambda_{i}\left(D^{2} v\right) v\right)^{2}}=\frac{\partial F}{\partial z}\left(D^{2} v, v\right) \quad \text { by }(3.6) .
\end{aligned}
$$

Consequently (3.5) transforms to read

$$
w_{t}=\frac{\partial F}{\partial r_{i j}}\left(D^{2} v, v\right) w_{x_{i} x_{j}}+2 \frac{\partial F}{\partial z}\left(D^{2} v, v\right) w \text { in } Q .
$$

In view of Lemma 2.1 this is a uniformly parabolic equation. As (3.3), (3.4) assert $w=0$ on the parabolic boundary of $Q$, we deduce $w=0$ everywhere within $Q$.

Owing to $(3.1),(3.2)$ we see that

$$
\Gamma \equiv\{(x, t) \in \bar{Q} \mid v=0\}
$$

is a smooth hypersurface in $\mathbf{R}^{n+1} \cap Q$ and each slice $\Gamma_{t} \equiv\{x \in V \mid(x, t) \in Q\}$ is a smooth hypersurface in $V$. 
Theorem 3.2. The surfaces $\left\{\Gamma_{t}\right\}_{0 \leq t \leq t_{0}}$ comprise a classical motion by mean curvature starting from $\Gamma_{0}$.

Proof. For each fixed $t$ in $\left[0, t_{0}\right], \nu \equiv D v$ is a normal unit vector field to $\Gamma_{t}$ in $\mathbf{R}^{n}$. In addition the PDE (1.13) implies

$$
v_{t}=\Delta v=\operatorname{div}(\nu) \text { on } \Gamma_{t} .
$$

However

$$
\operatorname{div}(\nu)=-(n-1) H=-\left(\kappa_{1}+\cdots+\kappa_{n-1}\right),
$$

where $\kappa_{1}, \ldots, \kappa_{n-1}$ are the principal curvatures of $\Gamma_{t}$ computed with respect to $\nu$ and

$$
H=\frac{1}{n-1}\left(\kappa_{1}+\cdots+\kappa_{n-1}\right)
$$

is the mean curvature.

Now fix $0 \leq t \leq t_{0}, x \in \Gamma_{t}$, and evolve the point $x$ according to the nonautonomous ODE

$$
\left\{\begin{array}{l}
\dot{x}(s)=-[\operatorname{div}(\nu) \nu](x(s), s) \quad(s>t) \\
x(t)=x
\end{array}\right.
$$

Then

$$
\begin{aligned}
\frac{d}{d s} v(x(s), s) & =-[(D v \cdot \nu) \operatorname{div}(\nu)](x(s), s)+v_{t}(x(s), s) \\
& =-\operatorname{div}(\nu)+v_{t}=0 .
\end{aligned}
$$

Thus

$$
v(x(s), s)=0 \quad(s>t),
$$

and this implies $\left\{\Gamma_{t}\right\}_{0 \leq t \leq t_{0}}$ is evolving by mean curvature.

\section{INSTANTANEOUS TEARING APART}

As a further application of the PDE (1.7)-(1.9) we prove in this section that two distinct smooth surfaces evolving by mean curvature must instantly tear completely apart, even if they initially touched on a large set.

More precisely, suppose as above $\Gamma_{0}$ is the smooth connected boundary of the bounded open set $U \subset \mathbf{R}^{n}$. Assume also that $\widehat{\Gamma}_{0}$ is the smooth connected boundary of a bounded open subset $\widehat{U} \subset \mathbf{R}^{n}$ and that

$$
\widehat{\Gamma}_{0} \subset \bar{U} \text {. }
$$

Thus the surface $\widehat{\Gamma}_{0}$ lies within the closed region $\bar{U}$ bounded by $\Gamma_{0}$. Suppose further that

$$
\widehat{\Gamma}_{0} \neq \Gamma_{0} .
$$

Choose next $t_{0}>0$ so small that the classical evolutions $\left\{\Gamma_{t}\right\}$ and $\left\{\widehat{\Gamma}_{t}\right\}$ starting at $\Gamma_{0}$ and $\widehat{\Gamma}_{0}$, respectively, exist at least for times $0 \leq t \leq t_{0}$.

Theorem 4.1. We have

$$
\Gamma_{t} \cap \widehat{\Gamma}_{t}=\varnothing \text { for } 0<t \leq t_{0} .
$$

We see therefore that the two evolutions by mean curvature instantly completely "tear apart". The point is that the PDE describing evolution by mean 
curvature is "uniformly parabolic along the surface" and thus admits infinite propagation speed for disturbances. See $[3, \S 8]$ for some geometric consequences.

Proof. 1. Fix $\delta_{0}$ so small that the signed distance function

is smooth in

$$
d(x, t) \equiv\left\{\begin{aligned}
-\operatorname{dist}\left(x, \Gamma_{t}\right) & \text { if } x \in U_{t}, \\
\operatorname{dist}\left(x, \Gamma_{t}\right) & \text { if } x \in \mathbf{R}^{n}-U_{t}
\end{aligned}\right.
$$

$$
Q \equiv\left\{(x, t) \mid-\delta_{0} \leq d(x, t) \leq \delta_{0}, 0 \leq t \leq t_{0}\right\} .
$$

(Here $U_{t}$ is the bounded region enveloped by $\Gamma_{t}$.) As above we have

$$
d_{t}=F\left(D^{2} d, d\right) \text { in } Q .
$$

Similarly the signed distance

$$
\hat{d}(x, t) \equiv\left\{\begin{aligned}
-\operatorname{dist}\left(x, \widehat{\Gamma}_{t}\right) & \text { if } x \in \widehat{U}_{t}, \\
\operatorname{dist}\left(x, \widehat{\Gamma}_{t}\right) & \text { if } x \in \mathbf{R}^{n}-\widehat{U}_{t}
\end{aligned}\right.
$$

is smooth in $Q$, provided $\delta_{0}, t_{0}>0$ are sufficiently small, and $\widehat{\Gamma}_{0}$ is close enough to $\Gamma_{0}$. (We denote by $\widehat{U}_{t}$ the region bounded by $\widehat{\Gamma}_{t}$.) Also

$$
\hat{d}_{t}=F\left(D^{2} \hat{d}, \hat{d}\right) \text { in } Q
$$

as well.

2. Now since (4.1) holds, we have $\widehat{\Gamma}_{t} \subseteq \bar{U}_{t}$ for each $0 \leq t \leq t_{0}$. Thus $\hat{d} \geq d$ in $Q$.

3. Set $w \equiv \hat{d}-d$. Then

$$
w \geq 0 \text { in } Q, \quad w \neq 0 \text { on } Q \cap\{t=0\},
$$

and from (4.4), (4.5) it follows that

$$
w_{t}=a_{i j} w_{x_{i} x_{j}}+c w \text { in } Q
$$

for

and

$$
a_{i j} \equiv \int_{0}^{1} \frac{\partial F}{\partial r_{i j}}\left(t D^{2} \hat{d}+(1-t) D^{2} d, t \hat{d}+(1-t) d\right) d t
$$

$$
c \equiv \int_{0}^{1} \frac{\partial F}{\partial z}\left(t D^{2} \hat{d}+(1-t) D^{2} d, t \hat{d}+(1-t) d\right) d t .
$$

According to Lemma 2.1 the coefficients $\left(\left(a_{i j}\right)\right)$ are uniformly positive definite if $\delta_{0}, t_{0}$ are small enough and $\widehat{\Gamma}_{0}$ is close enough to $\Gamma_{0}$. From (4.6), (4.7) and the strong maximum principle we deduce that

$$
w>0 \text { in the interior of } Q \text {. }
$$

Assertion (4.3) follows.

4. In the general case that $\widehat{\Gamma}_{0}$ verifies (4.1), (4.2), but is not necessarily everywhere close to $\Gamma_{0}$, we interpolate a smooth surface $\widetilde{\Gamma}_{0}$ between $\Gamma_{0}$ and $\widehat{\Gamma}_{0}$, so that $\widetilde{\Gamma}_{0}$ satisfies $(4.1),(4.2)$ and is close to $\Gamma_{0}$. Then, by steps $1-3$,

$$
\widetilde{\Gamma}_{t} \cap \Gamma_{t}=\varnothing
$$

for all small $t>0,\left\{\widetilde{\Gamma}_{t}\right\}$ denoting the classical evolution from $\widetilde{\Gamma}_{0}$. As $\tilde{\Gamma}_{t}$ lies within the region bounded by $\Gamma_{t}$ and $\widehat{\Gamma}_{t}$ lies in the region bounded by $\tilde{\Gamma}_{t}$, assertion (4.3) follows in this case as well. 


\section{REFERENCES}

1. L. A. Caffarelli, L. Nirenberg, and J. Spruck, The Dirichlet problem for nonlinear second order elliptic equations. III: Functions of the eigenvalues of the Hessian, Acta Math. 155 (1985), 261-301.

2. Y.-G. Chen, Y. Giga, and S. Goto, Uniqueness and existence of viscosity solutions of generalized mean curvature flow equations, J. Differential Geometry 33 (1991), 749-786.

3. L. C. Evans and J. Spruck, Motion of level sets by mean curvature. I, J. Differential Geometry 33 (1991), 635-681.

4. M. Gage and R. S. Hamilton, The heat equation shrinking convex plane curves, J. Differential Geom. 23 (1986), 69-96.

5. R. S. Hamilton, Three manifolds with positive Ricci curvature, J. Differential Geom. 17 (1982), 255-306.

6. O. A. Ladyzhenskaja, V. A. Solonnikov, and N. N. Ural'tseva, Linear and quasilinear equations of parabolic type, Amer. Math. Soc., Providence, R.I., 1968.

Department of Mathematics, University of California, Berkeley, California 94720

Department of Mathematics, University of Massachusetts, Amherst, Massachusetts 01003 